\title{
Clinical Outcomes of Transcatheter Aortic Valve Replacement in Nonagenarians: A Systematic Review and Meta-Analysis
}

\author{
Yan Liu, Yu Du, Mingjie Fu, Yue Ma, Deguang Wang, Jinglin Zhang, Wei Liu (D, \\ Yingxin Zhao, and Yujie Zhou \\ Department of Cardiology, 12th ward, Beijing Anzhen Hospital, Capital Medical University, Beijing Institute of Heart Lung and \\ Blood Vessel Disease, Beijing Key Laboratory of Precision Medicine of Coronary Atherosclerotic Disease, \\ Clinical Center for Coronary Heart Disease, Beijing 100029, China \\ Correspondence should be addressed to Yujie Zhou; azzyj12@163.com
}

Received 6 November 2018; Accepted 11 February 2019; Published 24 February 2019

Academic Editor: Andrea Rubboli

Copyright $\odot 2019$ Yan Liu et al. This is an open access article distributed under the Creative Commons Attribution License, which permits unrestricted use, distribution, and reproduction in any medium, provided the original work is properly cited.

\begin{abstract}
Objectives. To compare the incidence of mortality and complications between nonagenarians and younger patients undergoing transcatheter aortic valve replacement (TAVR). Background. TAVR has become an alternative treatment for nonagenarian patients with severe aortic stenosis. Previous studies have reported conflicting results regarding the clinical outcomes between nonagenarians and younger patients who underwent TAVR. Methods. We searched PubMed, EMBASE, and Cochrane Library databases with predefined criteria from the inception dates to July 8, 2018. The primary clinical endpoint was 30-day and 1-year all-cause mortalities. Secondary outcomes were considered the rates of stroke, myocardial infarction, any bleeding, any acute kidney injury, any vascular complications, new pacemaker implantation, and conversion to surgical aortic valve replacement. Results. A total of 5 eligible studies with 25,371 patients were included in this meta-analysis. Compared with younger patients who underwent TAVR, nonagenarians had a significantly higher mean Society of Thoracic Surgeons score (STS score) (MD, 2.80; 95\%CI: 2.58, 3.30; P<0.00001) and logistic European System for Cardiac Operative Risk Evaluation (logistic EuroSCORE) (MD, $2.72 ; 95 \% \mathrm{CI}: 1.01,4.43 ; \mathrm{P}=0.002)$. Nonagenarians were associated with significantly higher 30 -day mortality ( $6.2 \%$ vs. $3.7 \%$; OR, 1.73 ; $95 \% \mathrm{CI}: 1.49,2.00)$ and 1-year mortality $(15.5 \%$ vs. $11.8 \%$; OR, 1.39 ; $95 \% \mathrm{CI}: 1.26,1.53)$, without significant statistical heterogeneity. Nonagenarians were associated with significantly increased rates of major or life-threatening bleeding, vascular complications and stroke of $20 \%, 35 \%$, and $32 \%$, respectively. There were no significant differences in the rate of myocardial infarction, stage 2 or 3 acute kidney injury, new pacemaker implantation, or conversion to surgical aortic valve replacement. Conclusions. Nonagenarians showed worse clinical outcomes than younger patients after TAVR, while the incidence of mortality was acceptable. TAVR remains an option for nonagenarian patients with severe aortic stenosis and should be comprehensively evaluated by the heart valve team.
\end{abstract}

\section{Introduction}

As forecasted by Kontis et al., the life expectancy in industrialized countries would break the 90 -year barrier by 2030 [1]. Since the prevalence of aortic stenosis is increasing with lifespan, $[2,3]$ it is urgent to address the management of aortic stenosis (AS) for nonagenarians.

Since 2002, when the first transcatheter aortic valve replacement (TAVR) was carried out by Cribier, [4] it has become an alternative treatment for intermediate- to highrisk patients with severe AS [5, 6]. Several studies have shown no significant differences in the short- or long-term survival of nonagenarians between surgical aortic valve replacement (SAVR) and TAVR [7-9]. In consideration of frailty and inoperability, TAVR is a preferable approach for elderly patients.

However, previous studies have reported conflicting results regarding the clinical outcomes between nonagenarians and younger patients who underwent TAVR [10-14]. To further confirm the feasibility and safety of TAVR in nonagenarians, we performed this systematic review and meta-analysis to explore the short- to mid-term clinical outcomes of TAVR. 


\section{Methods}

This meta-analysis was performed based on the Cochrane Handbook for Systematic Reviews of Interventions [15] and is presented according to the MOOSE (Meta-analysis of Observational Studies in Epidemiology) guidelines [16].

2.1. Search Strategy. We searched PubMed, EMBASE, and Cochrane Library databases for the current literature. The following search terms were used: $((()$ ("transcatheter aortic valve replacement"[mesh]) OR transcatheter aortic valve implantation) OR TAVR) OR TAVI)) AND (((“Aged, 80 and over"[mesh]) OR 90 years) OR Nonagenarian*). No language, publication date, or publication status restrictions were imposed. The last search was performed on July 8, 2018. Two investigators (YL and YD) performed the initial search separately, deleted duplicate records, screened titles and abstracts for relevance, and identified relevant articles for further full-text assessment. Reference lists from these retrieved articles were manually screened to identify additional relevant studies.

2.2. Inclusion Criteria. Studies were selected based on the following inclusion criteria: (1) studies enrolling nonagenarian and younger patients undergoing TAVR in current clinical practices, (2) studies comparing clinical outcomes of nonagenarians to younger patients undergoing TAVR, and (3) studies in which the clinical endpoints and adverse events were diagnosed by the Valve Academic Research Consortium 2 definitions [17]. Conference abstracts, reviews, comments, and editorials were excluded.

2.3. Data Extraction and Quality Assessment. Two investigators (YL and YD) independently extracted data (first author, country of origin, publication year, number of enrolled patients, and baseline patient characteristics) using a standardized data abstraction form. When the same patients were reported in several publications, only the largest study was used for the meta-analysis to avoid data duplication.

Two investigators (YL and YD) independently assessed the quality of selected studies based on the 9-star NewcastleOttawa Scale [18]. This scale rates studies based on eight criteria in three sources of bias. Disagreement was resolved by discussions and by consulting a third investigator (YJZ).

2.4. Clinical Endpoints. The primary clinical endpoint of interest was 30-day and 1-year all-cause mortality, and secondary outcomes were considered as the rates of stroke, myocardial infarction, any bleeding, any acute kidney injury, any vascular complications, new pacemaker implantation, and conversion to surgical aortic valve replacement. All definitions of clinical endpoints were based on the Valve Academic Research Consortium 2 definitions.

2.5. Statistical Analysis. The odds ratios (ORs) with $95 \%$ confidence intervals (CIs) for the endpoints were calculated from each study. Trial-specific ORs were combined with the Mantel-Haenszel fixed-effects model or with random effects model if heterogeneity was statistically significant or $\mathrm{I}^{2}>50 \%$. If no events were reported for one group in a comparison, a value of 0.5 was added to both groups for each of these studies. Trials with no events in both groups were not included in the meta-analysis when the ORs were calculated.

The presence of heterogeneity among studies was evaluated with the Cochran $\mathrm{Q}$ chi-squared test, with $\mathrm{P}<0.10$ considered to indicate statistical significance, and the $\mathrm{I}^{2}$ test was used to evaluate inconsistencies. The $\mathrm{I}^{2}$ statistic is derived from the $Q$ statistic and describes the percentage of total variation across studies which is due to heterogeneity; values of $25 \%, 50 \%$, and $75 \%$ correspond to low, moderate, and high heterogeneity, respectively. The funnel plot was not drawn for outcomes due to the small number of studies included in this analysis.

We did not contact the authors of the included studies to obtain raw data. All analyses were performed using Review Manager version 5.3 (Copenhagen, Denmark; Cochrane Collaboration). All tests were two tailed, and $\mathrm{P}<0.05$ was considered significant.

\section{Results}

3.1. Study Characteristics and Quality Assessment. From the searches for meta-analysis, 5,238 potentially eligible studies were identified. Titles and abstracts of these studies were screened for inclusion. Full-texts of 44 studies were read, and 5 studies met the inclusion criteria (Figure 1) [10, 19-22]. The main characteristics of the included studies are described in Table 1(a). The current meta-analysis included 25,371 patients (3,929 in the nonagenarian group and 21,442 in the younger group). The mean Society of Thoracic Surgeons score (STS score), logistic European System for Cardiac Operative Risk Evaluation (logistic EuroSCORE), left ventricular ejection fraction (LVEF), mean pressure gradient, and medical history of the different studies are summarized in Tables 1(b) and 1(c). All trials reported that the clinical outcomes of interest varied from a 30-day to a 3-year follow-up period.

The assessment of quality is presented in Table 2 . The total score of the 5 observational studies was $>5$ according to the Newcastle-Ottawa Scale for risk of bias in observational studies, representing a low risk of bias.

3.2. Clinical Outcomes. Nonagenarians had a significantly higher mean STS score than younger patients (MD, 2.80; 95\% CI: 2.58, 3.30; $\mathrm{P}<0.00001)$, with low study heterogeneity $\left(\mathrm{P}=0.29 ; \mathrm{I}^{2}=20 \%\right.$, Figure 2$)$. Four studies analyzed the logistic EuroSCORE of patients, which was also higher in the nonagenarian group (MD, 2.72; 95\%CI: 1.01, 4.43; $\mathrm{P}=0.002$ ). No significant statistical heterogeneity was found among the studies (Figure 3).

There were 245 patients (6.2\%) with 30-day mortality reported among the nonagenarian group and 800 patients (3.7\%) among the younger group. The 30 -day mortality rates were significantly higher among nonagenarians (OR, 1.73; 95\%CI: 1.49, 2.00; $\mathrm{I}^{2}=0 \%$, Figure 4). Four studies reported 1year all-cause mortalities. The pooled average 1-year mortality was $12.4 \%$ and was $15.5 \%$ in the nonagenarian group and $11.8 \%$ 


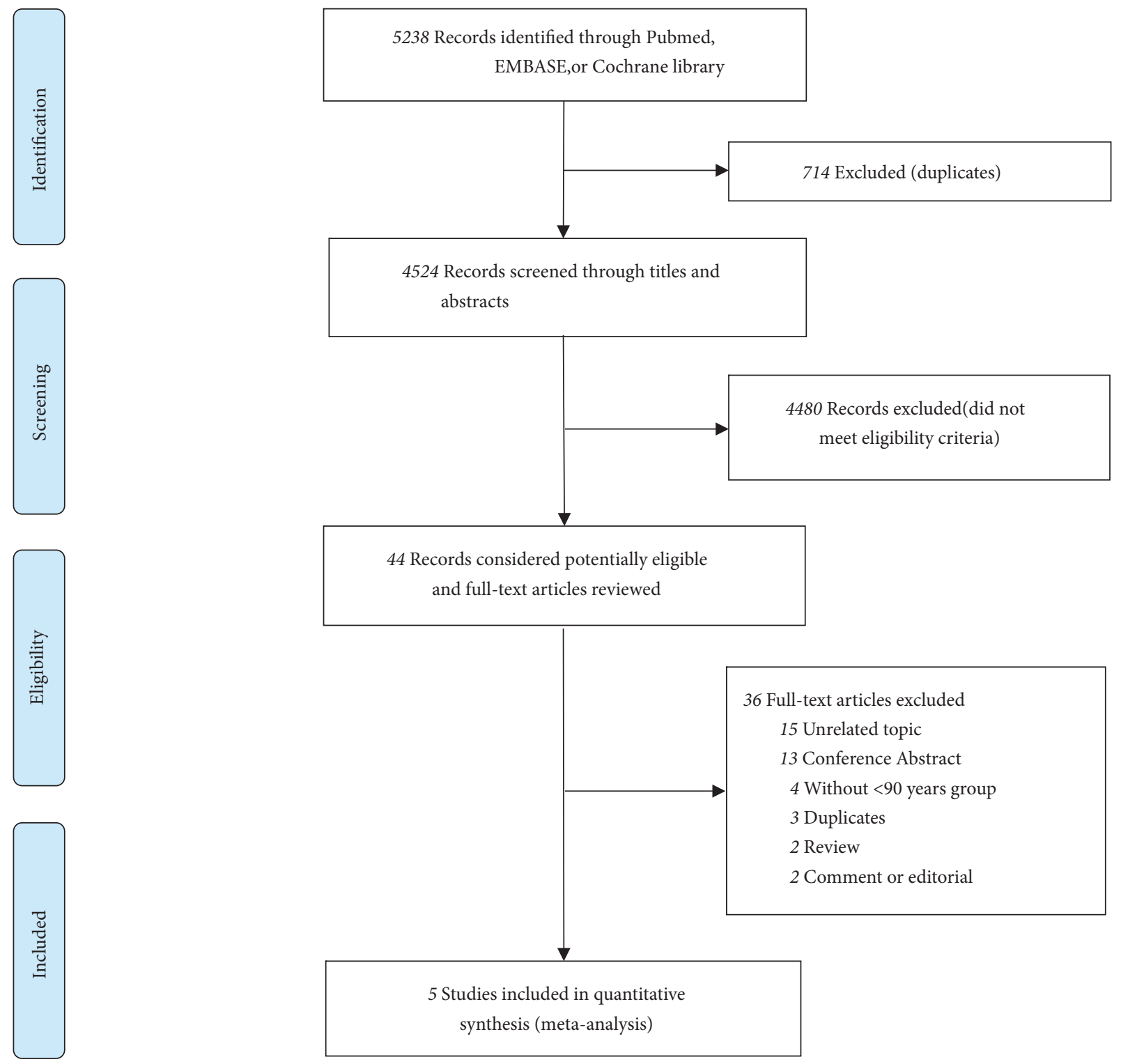

FIGURE 1: Flow diagram of study selection process.

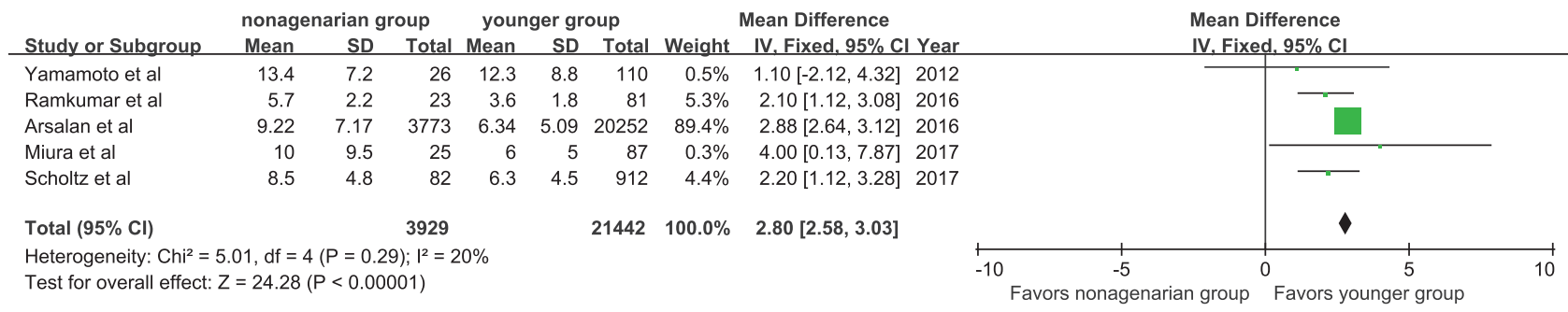

FIGURE 2: Forest plot of pooled analysis comparing Society of Thoracic Surgeons score of nonagenarians versus younger patients.

in the younger group. Nonagenarians were associated with a significantly higher 1-year mortality (OR, 1.39; $95 \% \mathrm{CI}: 1.26$, $1.53 ; \mathrm{I}^{2}=0 \%$, Figure 5 ).

The specific definitions of any bleeding, any acute kidney injury, and any vascular complications are shown in
Table 3. Major or life-threatening bleeding was reported in 313 patients $(8.1 \%)$ in the nonagenarian group and in 1,405 patients $(6.8 \%)$ in the younger group. Vascular complications were reported in 135 patients (3.43\%) in the nonagenarian group and $553(2.6 \%)$ in the younger group. Nonagenarians 


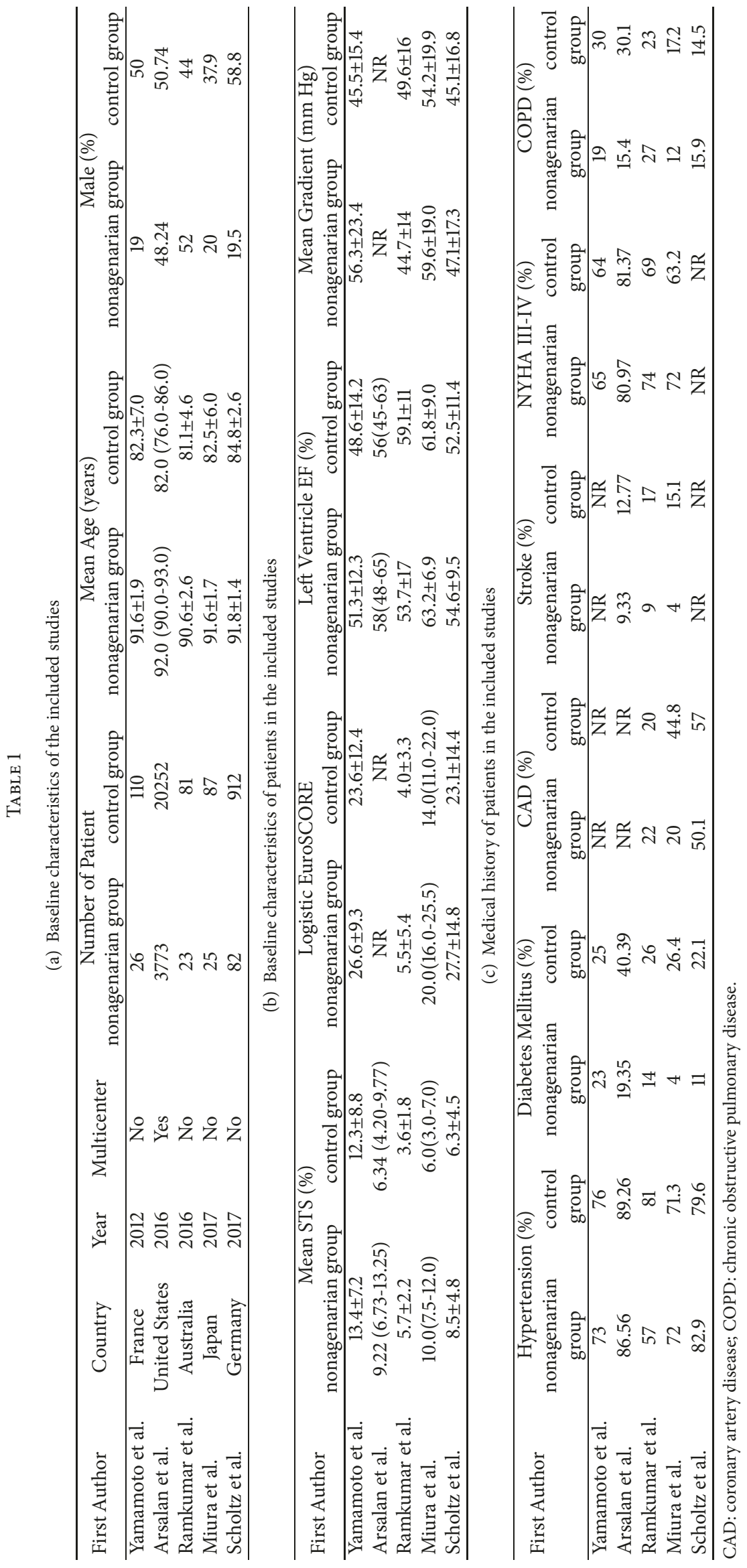




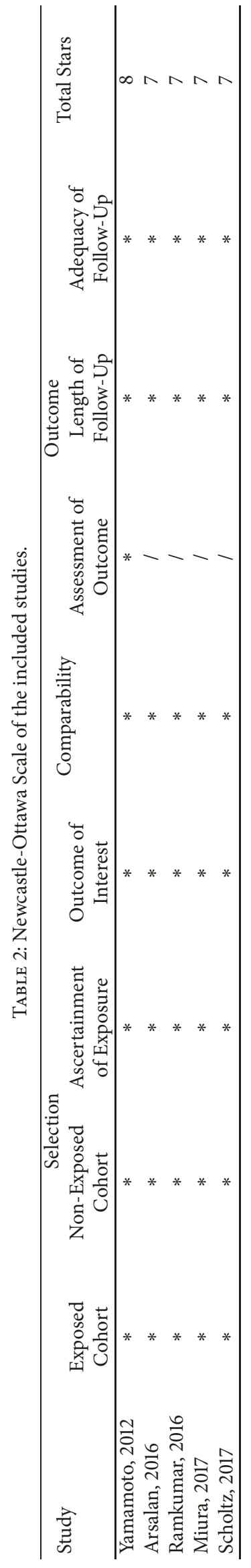


TABLE 3: The specific definitions of bleeding, acute kidney injury, and vascular complications.

\begin{tabular}{lccc}
\hline First Author & Bleeding & Acute kidney injury & vascular complications \\
\hline Yamamoto et al. & Major, life-threatening & Stage 2 or 3 & Major \\
Arsalan et al. & Major & New requirement for dialysis & Major, minor \\
Ramkumar et al. & Major & Stage 2 or 3 & Major \\
Miura et al. & Life-threatening & Stage 2 or 3 & Major, minor \\
Scholtz et al. & NR & NR & No specified \\
\hline
\end{tabular}

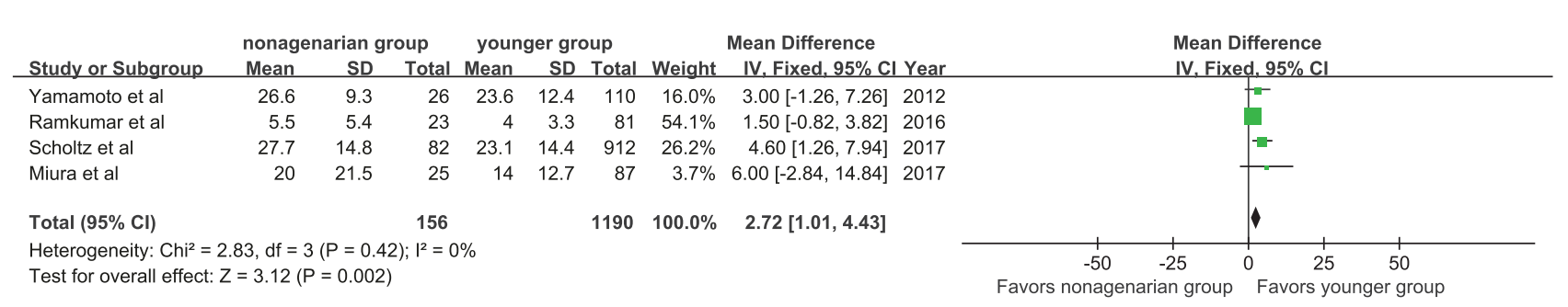

FIGURE 3: Forest plot of pooled analysis comparing logistic European System for Cardiac Operative Risk Evaluation of nonagenarians versus younger patients.

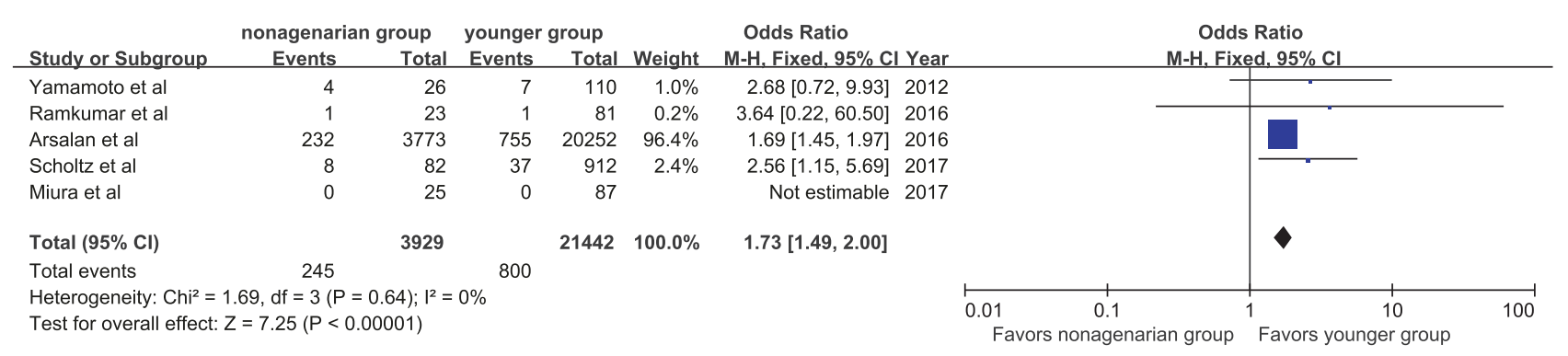

FIGURE 4: Forest plot of pooled analysis comparing 30-day mortality of nonagenarians versus younger patients.

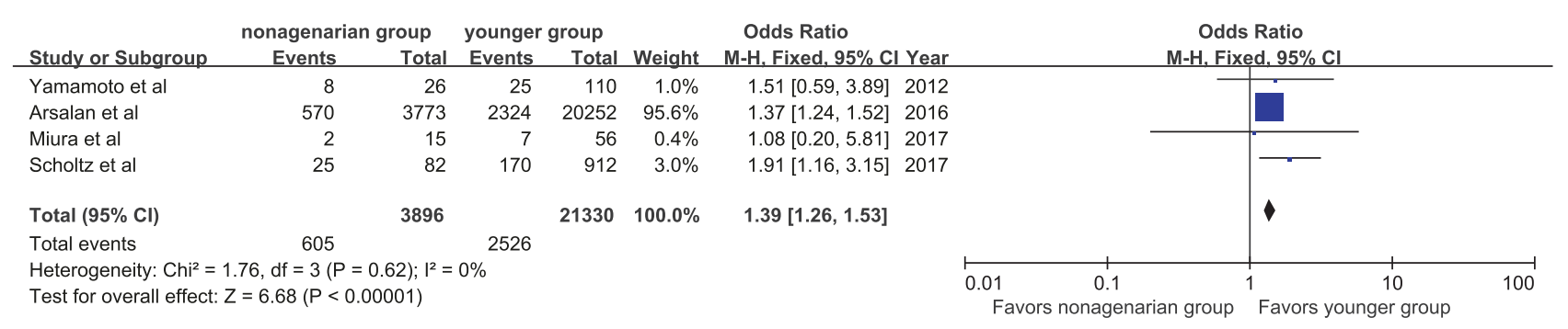

FIGURE 5: Forest plot of pooled analysis comparing 1-year mortality of nonagenarians versus younger patients.

were associated with a significantly higher rate of major or life-threatening bleeding (OR, 1.20; 95\%CI: 1.05, 1.36; $\mathrm{I}^{2}=0 \%$, Figure 6) and vascular complications (OR, 1.35; 95\%CI: 1.11, $1.64 ; \mathrm{I}^{2}=4 \%$, Figure 7 ). In addition, we observed that nonagenarians had a higher risk of stroke than younger patients, with evidence of low heterogeneity (OR, 1.32; 95\%CI: 1.08, $1.62 ; \mathrm{I}^{2}=1 \%$, Figure 8).

There were no significant differences in the rate of myocardial infarction (OR, 1.09; 95\%CI: 0.80, 1.49; $\mathrm{I}^{2}=0 \%$ ), stage 2 or 3 acute kidney injury (OR, 0.84; 95\%CI: 0.65, 1.10; $\mathrm{I}^{2}=0 \%$ ), new pacemaker implantation (OR, 0.97; 95\%CI: 0.59, $1.59 ; \mathrm{I}^{2}=0 \%$ ), or conversion to surgical aortic valve replacement (OR, 2.03; 95\%CI: 0.53, 7.77; $\mathrm{I}^{2}=0 \%$ ) (Figures 9-12).

\section{Discussion}

To the best of our knowledge, this is the first comprehensive review of the current literature comparing the clinical outcomes of TAVR between nonagenarian and younger patients in a meta-analytic approach. In the results reported here, nonagenarians, with a higher mean STS score and logistic EuroSCORE, had an increased 30-day and 1-year postoperative all-cause mortalities compared with the younger group. In addition, the rates of major or life-threatening bleeding, vascular complications and stroke were also higher in nonagenarians. Furthermore, no significant differences were observed in the rates of myocardial infarction, stage 2 or 3 acute kidney injury, new pacemaker implantation, and 


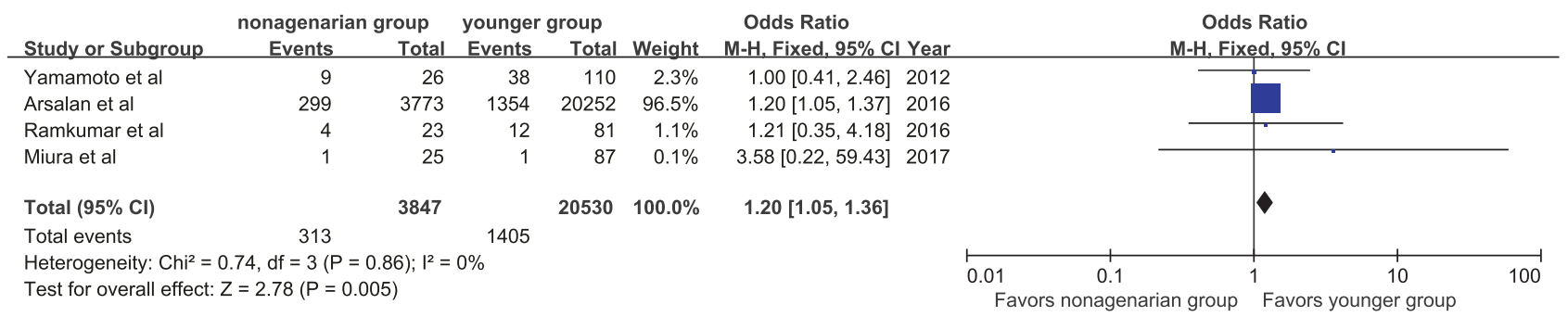

FIGURE 6: Forest plot of pooled analysis comparing the rates of major or life-threatening bleeding in nonagenarians versus younger patients.

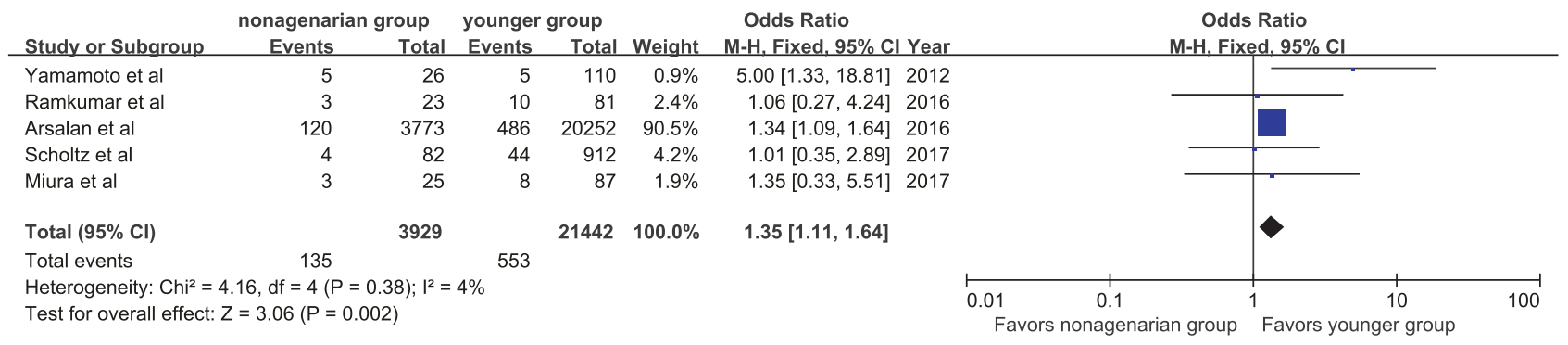

FIGURE 7: Forest plot of pooled analysis comparing the rates of vascular complication in nonagenarians versus younger patients.

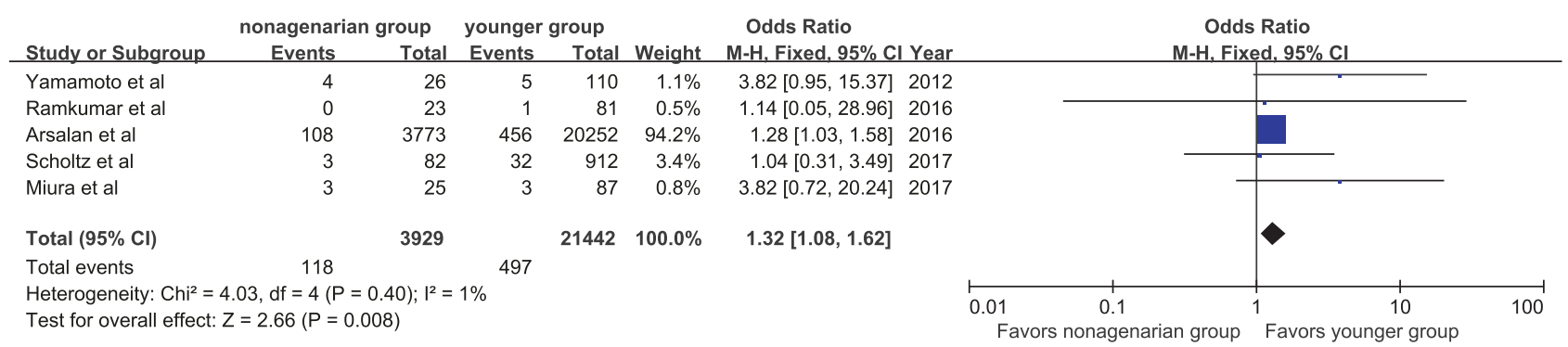

FIGURE 8: Forest plot of pooled analysis comparing the rates of stroke in nonagenarians versus younger patients.

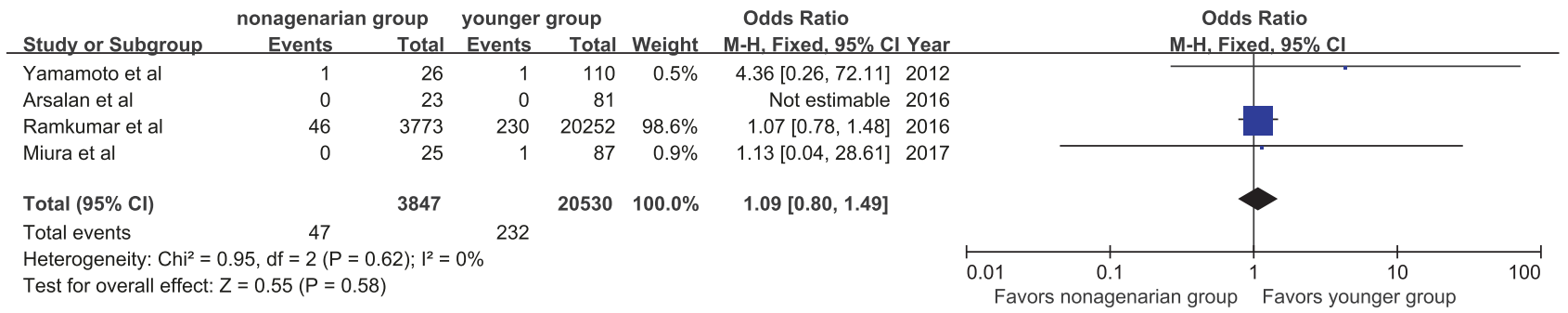

FIGURE 9: Forest plot of pooled analysis comparing the rates of myocardial infarction in nonagenarians versus younger patients.

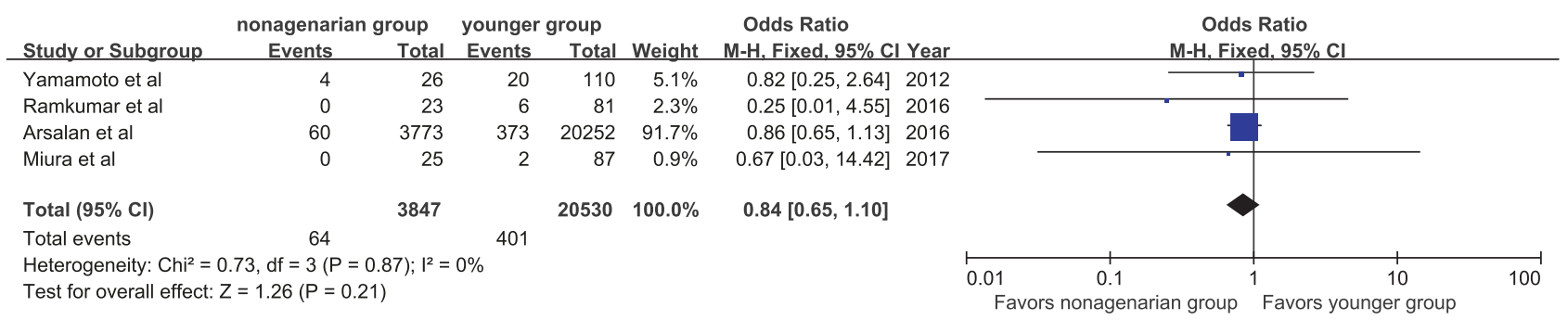

FIGURE 10: Forest plot of pooled analysis comparing the rates of stage 2 or 3 acute kidney injury in nonagenarians versus younger patients. 


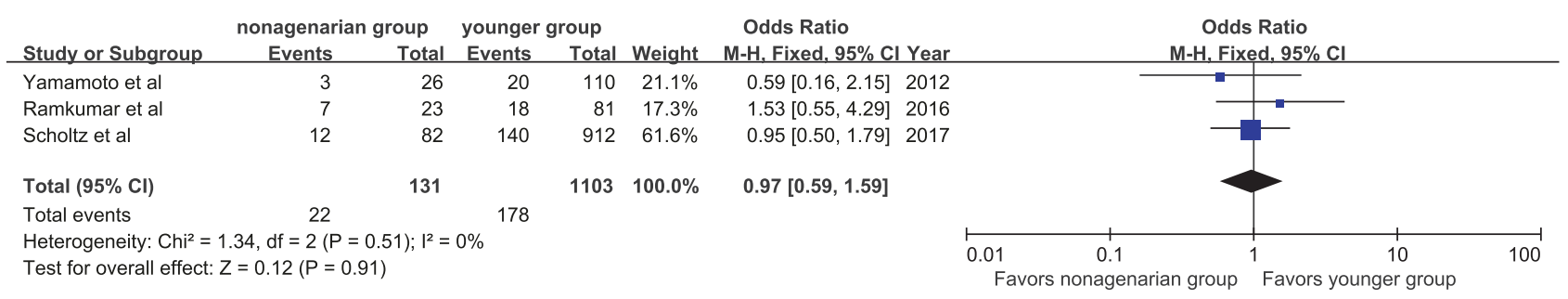

FIGURE 11: Forest plot of pooled analysis comparing the rates of new pacemaker implantation in nonagenarians versus younger patients.

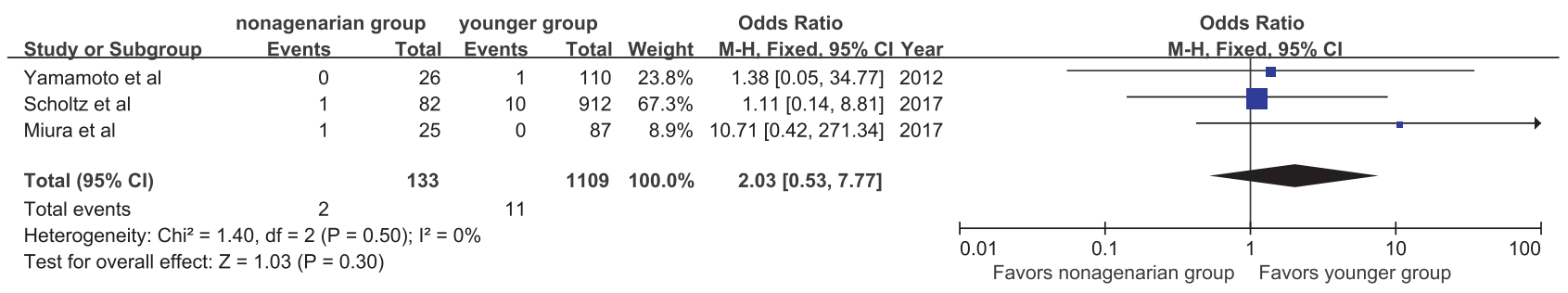

FIGURE 12: Forest plot of pooled analysis comparing the rates of conversion to aortic valve replacement in nonagenarians versus younger patients.

conversion to surgical aortic valve replacement between the two groups.

Although the mortality rate for nonagenarians in our study remained higher than for younger patients, considering life expectations, comparison of medical treatment, and quality of life, the mortality rate may be acceptable. It was reported in nonagenarians that age alone accounted for a predicted logistic EuroSCORE mortality risk of $6.55 \%$ for male patients, and for female patients, this risk rises to $8.89 \%$ without any other preoperative risk factors [23]. Similarly, our results showed that nonagenarians had a significantly higher logistic EuroSCORE than younger patients (MD, 2.72; 95\%CI: 1.01, 4.43). Bernal et al. indicated that approximately one-third of nonagenarians with severe aortic stenosis have few comorbidities [24]. Therefore, in a particular population, patients at a very advanced age could be the primary factor making nonagenarians have a high risk for surgery. Furthermore, Bernal et al. observed that nonagenarians who underwent conservative management tended to have a higher 1-year mortality than those who underwent TAVR (58\% vs. $40.7 \%, \mathrm{P}=0.097$ ) [24]. In our meta-analyses, the pooled postoperative 30-day and 1-year all-cause mortalities of nonagenarians was $6.2 \%$ and $15.5 \%$, respectively. Despite the higher mortality than younger patients, TAVR could be a better option for severe AS nonagenarians.

Manolis et al. demonstrated that, in nonagenarians who underwent TAVR, bleeding and vascular complications ranged from $9 \%$ to $34 \%$ (average 16\%), and stroke risk ranged from $2 \%$ to $18 \%$ (average $3-4 \%$ ) [25]. Similarly, in this meta-analysis, we indicated that nonagenarians were associated with significantly increased rates of major or lifethreatening bleeding, vascular complications and stroke of $20 \%, 35 \%$, and $32 \%$, respectively. The higher incidence of vascular complications may be attributed to the higher rates of transfemoral access. Another possible reason could be the higher rates of vascular calcification [26] in the "oldest old" population. The higher incidence of stroke and major or life-threatening bleeding may be attributed to the higher prevalence of atrial fibrillation [11] and antiplatelet therapy after TAVR [27], respectively.

In the general population, life expectancy in nonagenarians is 2.5 and 3.5 years for men and women, respectively [23]. The rate of 1-year mortality in nonagenarians was 19.3\% [28]. Accordingly, we should focus on the improvement of quality of life and try to add life to their years instead of years to their life [29]. Stanska et al. showed that the quality of life in elderly patients was significantly improved at the one-month followup after TAVR [30]. Mack et al. indicated that nonagenarians undergoing TAVR have an improvement in quality of life (47.2 to 74.0, $\mathrm{P}=0.051$ ), as measured by the Kansas City Cardiomyopathy Questionnaire (KCCQ) [7]. Arsalan et al. reported that nonagenarians have similar KCCQ scores with younger patients 1 year after TAVR [10].

Nonagenarians referred for TAVR should be evaluated carefully by the heart valve team composited of cardiovascular surgeons, interventional cardiologists, imaging specialists, cardiovascular anesthesiologists, and cardiovascular nursing professionals [31]. Generally, we use the STS score and logistic EuroSCORE to assess the risk of TAVR, and neither of these scores includes the specific geriatric conditions, which tend to generate a considerable impact on prognosis in elderly patients. Okoh et al. indicated that frailty status is independently associated with increased mortality after TAVR (hazard ratio: 1.84; 95\%CI: 1.06-3.17; $\mathrm{P}=0.028$ ) [32]. Multidimensional Geriatric Assessment (MGA) is a diagnostic process that determines the medical and functional resources and problems of elderly patients. According to Stortecky et al., risk prediction of TAVR can be improved by adding MGA-based information to global risk scores [33]. Furthermore, in nonagenarians, transapical TAVR was associated with a significantly higher risk of early mortality compared with transfemoral TAVR [34-37]. The procedure 
should be comprehensively evaluated by the heart valve team.

This study has several limitations. First, all of the eligible studies were observational studies, and the results may have been affected by unmeasured confounding variables. Second, due to the limited number of studies included in this analysis, we did not conduct sensitivity and meta-regression analysis for outcomes. Third, our analysis did not include individual patient data. Finally, these data are mostly from highly experienced TAVR centers and may not be generalizable to other hospitals with less experience.

In conclusion, the results reported here suggest that nonagenarians showed higher short- to mid-term mortalities and higher rates of major or life-threatening bleeding, vascular complications and stroke compared to younger patients. However, the rate of mortality in nonagenarians is potentially acceptable. TAVR remains an optional therapy for nonagenarian patients, which should be comprehensively evaluated by the heart valve team.

\section{Abbreviations}

TAVR:

Transcatheter aortic valve replacement

STS score: Thoracic Surgeons score

logistic EuroSCORE: Logistic European System for Cardiac Operative Risk Evaluation

AS: Aortic stenosis

SAVR:

MOOSE: Surgical aortic valve replacement Meta-analysis of Observational Studies in Epidemiology

OR: Odds ratio

CI: Confidence interval

LVEF: KCCQ: Left ventricular ejection fraction Kansas City Cardiomyopathy Questionnaire

MGA: Multidimensional Geriatric Assessment

CAD: COPD: Coronary artery disease Chronic obstructive pulmonary disease.

\section{Data Availability}

The data supporting this article are from previously reported studies and datasets, which have been cited. All relevant data are within the article.

\section{Conflicts of Interest}

The authors declare that they have no conflicts of interest.

\section{Acknowledgments}

This work was supported by the Beijing Municipal Administration of Hospitals Clinical Medicine Development of Special Funding Support (code: ZYLX201303, XMLX201601), the grant from National Key Research and Development Program of China (2017YFC0908800), and Beijing Municipal Administration of Hospitals' Ascent Plan (Code: DFL20150601) and Mission plan (Code: SML20180601).

\section{References}

[1] V. Kontis, J. E. Bennett, C. D. Mathers, G. Li, K. Foreman, and M. Ezzati, "Future life expectancy in 35 industrialised countries: projections with a Bayesian model ensemble," The Lancet, vol. 389, no. 10076, pp. 1323-1335, 2017.

[2] F. Z. Liu, Y. M. Xue, H. T. Liao, X. Z. Zhan, H. M. Guo, H. L. Huang et al., "Five-year epidemiological survey of valvular heart disease: changes in morbidity, etiological spectrum and management in a cardiovascular center of Southern China," Journal of Thoracic Disease, vol. 6, no. 12, pp. 1724-1730, 2014.

[3] N. Rezzoug, B. Vaes, C. de Meester et al., "The clinical impact of valvular heart disease in a population-based cohort of subjects aged 80 and older," BMC Cardiovascular Disorders, vol. 16, no. 1, p. 7, 2016.

[4] A. Cribier, H. Eltchaninoff, A. Bash et al., "Percutaneous transcatheter implantation of an aortic valve prosthesis for calcific aortic stenosis: first human case description," Circulation, vol. 106, no. 24, pp. 3006-3008, 2002.

[5] R. A. Nishimura, C. M. Otto, R. O. Bonow et al., "2017 AHA/ACC focused update of the 2014 AHA/ACC guideline for the management of patients with valvular heart disease: a report of the american college of cardiology/american heart association task force on clinical practice guidelines," Circulation, vol. 135, no. 25, pp. el159-e1195, 2017.

[6] H. Baumgartner, V. Falk, J. J. Bax, M. De Bonis, C. Hamm, P. J. Holm et al., "2017 ESC/EACTS Guidelines for the management of valvular heart disease," European Heart Journal, vol. 38, no. 36, pp. 2739-2791, 2017.

[7] M. C. Mack, M. Szerlip, M. A. Herbert et al., "Outcomes of treatment of nonagenarians with severe aortic stenosis," The Annals of Thoracic Surgery, vol. 100, no. 1, Article ID 27989, pp. 74-80, 2015.

[8] C. R. Smith, M. B. Leon, M. J. Mack, D. C. Miller, J. W. Moses, L. G. Svensson et al., "Transcatheter versus surgical aortic-valve replacement in high-risk patients," The New England Journal of Medicine, vol. 364, no. 23, pp. 2187-2198, 2011.

[9] T. Murashita, K. L. Greason, R. M. Suri et al., "Aortic valve replacement for severe aortic valve stenosis in the nonagenarian patient," The Annals of Thoracic Surgery, vol. 98, no. 5, pp. 15931597, 2014.

[10] M. Arsalan, M. Szerlip, S. Vemulapalli et al., "Should transcatheter aortic valve replacement be performed in nonagenarians?: insights from the STS/ACC TVT registry," Journal of the American College of Cardiology, vol. 67, no. 12, pp. 1387-1395, 2016.

[11] Y. Abramowitz, T. Chakravarty, H. Jilaihawi et al., "Comparison of outcomes of transcatheter aortic valve implantation in patients $\geq 90$ years versus $<90$ years," American Journal of Cardiology, vol. 116, no. 7, pp. 1110-1115, 2015.

[12] R. O. Escárcega, N. C. Baker, M. J. Lipinski et al., "Clinical profiles and correlates of mortality in nonagenarians with severe aortic stenosis undergoing transcatheter aortic valve replacement," American Heart Journal, vol. 173, pp. 118-125, 2016.

[13] R. A. Meneguz-Moreno, A. N. Ferreira Neto, A. Ramos et al., "TCT-755 comparison of clinical profile and outcomes after 
transcatheter aortic valve replacement in nonagenarians versus younger patients," Journal of the American College of Cardiology, vol. 68, no. 18, p. B305, 2016.

[14] M. Yamamoto, G. Mouillet, K. Meguro et al., "Clinical results of transcatheter aortic valve implantation in octogenarians and nonagenarians: insights from the FRANCE-2 registry," The Annals of Thoracic Surgery, vol. 97, no. 1, pp. 29-36, 2014.

[15] J. P. T. Higgins and S. Green, Eds., Cochrane Handbook for Systematic Reviews of Interventions Version 5.1.0, The Cochrane Collaboration, 2011, https://training.cochrane.org/handbook.

[16] D. F. Stroup, J. A. Berlin, S. C. Morton et al., "Meta-analysis of observational studies in epidemiology: a proposal for reporting," Journal of the American Medical Association, vol. 283, no. 15, pp. 2008-2012, 2000.

[17] A. P. Kappetein, S. J. Head, P. Généreux et al., "Updated standardized endpoint definitions for transcatheter aortic valve implantation: the valve academic research consortium- 2 consensus document," Journal of the American College of Cardiology, vol. 60, no. 15, pp. 1438-1454, 2012.

[18] A. Stang, "Critical evaluation of the Newcastle-Ottawa scale for the assessment of the quality of nonrandomized studies in metaanalyses," European Journal of Epidemiology, vol. 25, no. 9, pp. 603-605, 2010.

[19] M. Yamamoto, K. Meguro, G. Mouillet et al., "Comparison of effectiveness and safety of transcatheter aortic valve implantation in patients aged $\geq 90$ years versus $<90$ years," American Journal of Cardiology, vol. 110, no. 8, pp. 1156-1163, 2012.

[20] S. Ramkumar, H. N. Rashid, S. Zaman et al., "Feasibility and clinical outcomes in nonagenarians undergoing transcatheter aortic valve replacement with the LOTUS valve," Journal of Geriatric Cardiology, vol. 13, no. 7, pp. 636-638, 2016.

[21] M. Miura, S. Shirai, Y. Uemura et al., "Early safety and efficacy of transcatheter aortic valve implantation for Asian nonagenarians (From KMH registry)," International Heart Journal, vol. 58, no. 6, pp. 900-907, 2017.

[22] S. Scholtz, Z. Dimitriadis, M. Vlachojannis et al., "Transcatheter aortic valve implantation in nonagenarians: procedural outcome and mid-term results," Heart, Lung and Circulation, vol. 27, no. 6, pp. 725-730, 2018.

[23] B. G. Levy Praschker, P. Leprince, N. Bonnet et al., "Cardiac surgery in nonagenarians: hospital mortality and long-term follow-up," Interactive CardioVascular and Thoracic Surgery, vol. 5, no. 6, pp. 696-699, 2006.

[24] E. Bernal, A. Ariza-Solé, A. Bayés-Genís et al., "Management of nonagenarian patients with severe aortic stenosis: the role of comorbidity," Heart, Lung and Circulation, vol. 27, no. 2, pp. 219226, 2018.

[25] A. S. Manolis and A. A. Manolis, "Transcatheter aortic valve implantation in nonagenarians: Selectively feasible or extravagantly futile?" Annals of Cardiothoracic Surgery, vol. 6, no. 5, pp. 524-531, 2017.

[26] F. Idoate, E. L. Cadore, A. Casas-Herrero et al., "Noncoronary vascular calcification, bone mineral density, and muscle mass in institutionalized frail nonagenarians," Rejuvenation Research, vol. 20, no. 4, pp. 298-308, 2017.

[27] H. Raheja, A. Garg, S. Goel et al., "Comparison of single versus dual antiplatelet therapy after TAVR: A systematic review and meta-analysis," Catheterization and Cardiovascular Interventions, vol. 92, no. 4, pp. 783-791, 2018.

[28] F. Formiga, A. Ferrer, J. Mascaró, D. Ruiz, C. Olmedo, and R. Pujol, "Predictive items of one-year mortality in nonagenarians.
The NonaSantfeliu study," Aging Clinical and Experimental Research, vol. 19, no. 4, pp. 265-268, 2007.

[29] T. Al-Atassi and M. Boodhwani, "Transcatheter aortic valve implantation in nonagenarians: Looking beyond survival," The Journal of Thoracic and Cardiovascular Surgery, vol. 150, no. 3, pp. 723-724, 2015.

[30] A. Stańska, D. Jagielak, M. Brzeziński et al., "Improvement of quality of life following transcatheter aortic valve implantation in the elderly: a multi-centre study based on the Polish national TAVI registry," Kardiologia Polska, vol. 75, no. 1, pp. 13-20, 2017.

[31] C. Writing, C. M. Otto, D. J. Kumbhani et al., "2017 ACC expert consensus decision pathway for transcatheter aortic valve replacement in the management of adults with aortic stenosis: a report of the american college of cardiology task force on clinical expert consensus documents," Journal of the American College of Cardiology, 2017.

[32] A. K. Okoh, D. Chauhan, N. Kang et al., “The impact of frailty status on clinical and functional outcomes after transcatheter aortic valve replacement in nonagenarians with severe aortic stenosis," Catheterization and Cardiovascular Interventions, vol. 90, no. 6, pp. 1000-1006, 2017.

[33] S. Stortecky, A. W. Schoenenberger, A. Moser et al., "Evaluation of multidimensional geriatric assessment as a predictor of mortality and cardiovascular events after transcatheter aortic valve implantation," JACC: Cardiovascular Interventions, vol. 5, no. 5, pp. 489-496, 2012.

[34] F. Biancari, P. D’Errigo, S. Rosato et al., “Transcatheter aortic valve replacement in nonagenarians: early and intermediate outcome from the OBSERVANT study and meta-analysis of the literature," Heart and Vessels, vol. 32, no. 2, pp. 157-165, 2017.

[35] M. O. Kayatta, V. H. Thourani, H. A. Jensen et al., "Outcomes for transcatheter aortic valve replacement in nonagenarians," The Annals of Thoracic Surgery, vol. 100, no. 4, pp. 1261-1267, 2015.

[36] C. McNeely, A. Zajarias, R. Robbs, S. Markwell, and C. M. Vassileva, "Transcatheter aortic valve replacement outcomes in nonagenarians stratified by transfemoral and transapical approach," The Annals of Thoracic Surgery, vol. 103, no. 6, pp. 1808-1814, 2017.

[37] V. H. Thourani, H. A. Jensen, V. Babaliaros et al., "Outcomes in nonagenarians undergoing transcatheter aortic valve replacement in the PARTNER-I trial," The Annals of Thoracic Surgery, vol. 100, no. 3, pp. 785-792, 2015. 


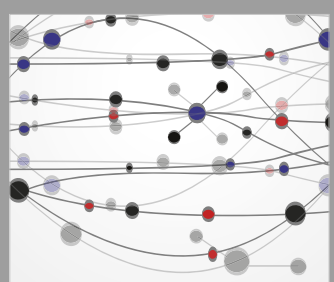

The Scientific World Journal
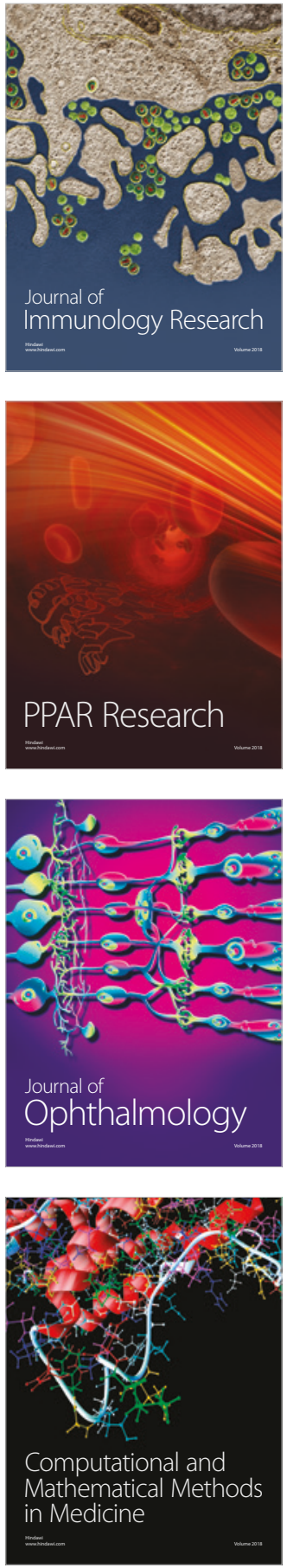

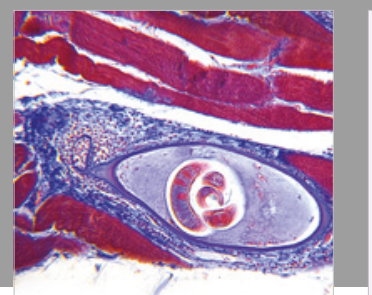

Gastroenterology Research and Practice

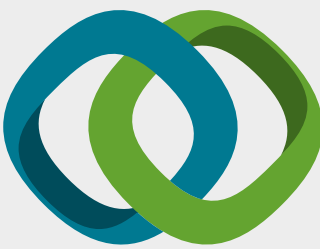

\section{Hindawi}

Submit your manuscripts at

www.hindawi.com
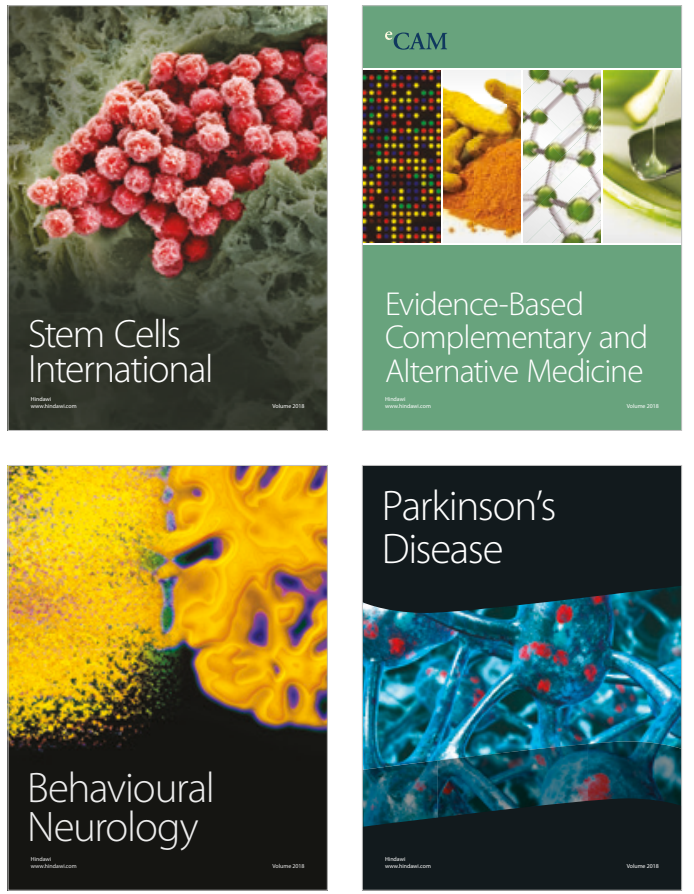

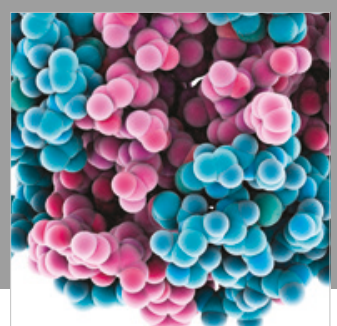

ournal of

Diabetes Research

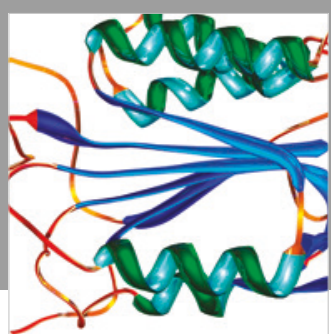

Disease Markers
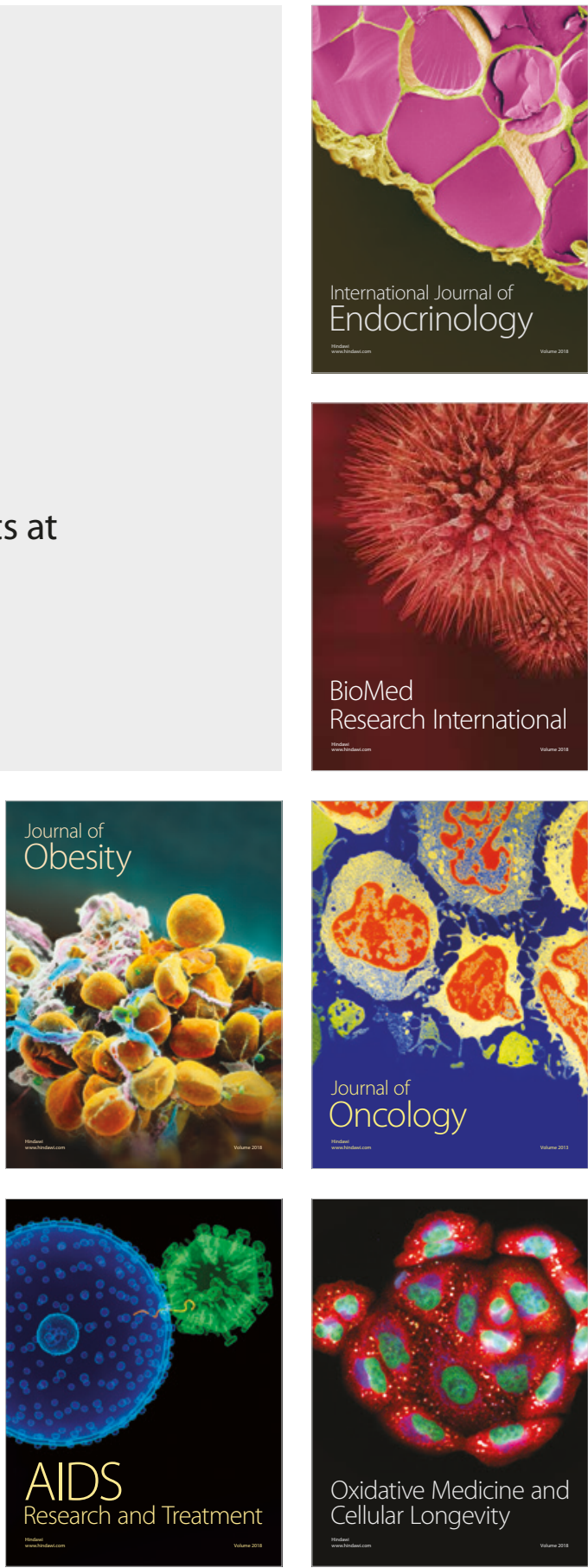